1 Associação de Pais e Amigos dos Excepcionais (Apae)- Florianópolis (SC), Brasil.

Orcid: https://orcid. org/0000-0002-93891679

licapanema@gmail.com

2 Universidade Federal de Santa Catarina (UFSC), Núcleo de Estudos da Terceira Idade (Neti) Florianópolis (SC), Brasil. Orcid: https://orcid. org/0000-0001-5117-

7234

jordelina.schier@ufsc.br

3 Universidade Federal de Santa Catarina (UFSC),

Laboratório de Pesquisas e Tecnologias em

Enfermagem, Cuidado em Saúde a Pessoas Idosas -

Florianópolis (SC), Brasil. Orcid: https://orcid. org/0000-0002-37634176

juliana.balbinot@ufsc.br

4 Universidade Federal de Santa Catarina (UFSC),

Laboratório de Pesquisas e Tecnologias em

Enfermagem, Cuidado em Saúde a Pessoas Idosas -

Florianópolis (SC), Brasil. Orcid: https://orcid. org/0000-0002-7140-

3427

karina.h@ufsc.br

5 Universidade Federal de Santa Catarina (UFSC),

Laboratório de Pesquisas e Tecnologias em

Enfermagem, Cuidado em

Saúde a Pessoas Idosas -

Florianópolis (SC), Brasil.

Orcid: https://orcid.

org/0000-0003-1451-

4566

franciscoreistristao@hotmail.

com

\section{Centro-dia: uma opção no atendimento da pessoa envelhecente com deficiência intelectual}

\author{
Center day: an option on attendance elder person with intellectual \\ disability
}

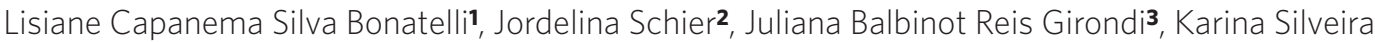
de Almeida Hammerschmidt ${ }^{4}$, Francisco Reis Tristão ${ }^{5}$

DOI: 10.1590/0103-1104201811810

RESUMO O estudo objetivou mapear serviços, fluxos de atendimento e organização do serviço do Centro-Dia das Associações de Pais e Amigos dos Excepcionais da grande Florianópolis para o atendimento da pessoa envelhecente com deficiência intelectual. Trata-se de pesquisa exploratório descritiva, com análise temática e referencial teórico de Feuerstein, realizada em 17 associações da grande Florianópolis, Santa Catarina. Evidenciou-se carência de implementação dos dispositivos legais referentes à pessoa com deficiência e idoso apontando necessidade de discussão e fortalecimento das políticas públicas na área da deficiência e envelhecimento, com vistas à qualificação do cuidado.

PALAVRAS-CHAVE Pessoas com deficiência. Envelhecimento. Centros comunitários para idosos. Enfermagem geriátrica.

ABSTRACT The study aimed to map out services, care services and organization of the Center-Day Service of Parents 'and Friends' Associations of the Greater Florianópolis to attend the person involved with intellectual disability. It is a descriptive exploratory research, with thematic analysis and theoretical reference of Feuerstein, performed in 17 associations of the great Florianópolis, Santa Catarina. There was a lack of implementation oflegal provisions regarding the disabled and elderly person, pointing out the need to discuss and strengthen public policies in the area of disability and aging, with a view to qualifying care.

KEYWORDS Disabled persons. Aging. Senior centers. Geriatric nursing: 


\section{Introdução}

Embora o Estatuto do Idoso ${ }^{1}$ saliente que a pessoa idosa compreende, cronologicamente, aquela com idade igual ou maior de 60 anos, estudiosos descrevem que as pessoas com deficiência intelectual apresentam o processo de envelhecimento acelerado, utilizando-se a idade de 35 anos, como marco do envelhecer, uma vez que reflete em diversas modificações funcionais e alto índice de demências, implicando na necessidade de cuidados específicos ${ }^{2}$.

Assim, proteger os direitos da pessoa com deficiência é uma preocupação mundial, sendo esta, disposta na Declaração dos Direitos das Pessoas Portadoras de Deficiências ${ }^{3}$, a qual solicita a adoção de medidas em planos nacionais e internacionais para o apoio e proteção desses direitos. Os dispositivos legais buscam garantir os direitos destas pessoas, sua proteção, saúde e qualidade de vida ${ }^{3}$.

A literatura sugere, que a maioria dos idosos deseja morar em sua própria casa, e a partir do momento que não mais conseguem se gerir, necessitam que algum membro da família assuma o cuidado ${ }^{4}$.

De acordo com a Politica Nacional do Idoso $^{5}$ e a Política Nacional de Promoção da Saúde 6 no caso da pessoa com deficiência, esse cuidado já acontece desde muito cedo, ainda na infância e intensifica-se na medida em que o processo de envelhecimento avança.

A Política Nacional do Idoso 5 aponta que 'centro-dia' é local destinado à permanência diurna do idoso dependente ou que possua deficiência temporária e necessite de assistência médica ou multiprofissional.

$\mathrm{O}$ 'centro-dia' é destinado àqueles com dependência parcial nas atividades de vida diária (auxílio na alimentação, vestuário, higiene pessoal, mobilidade) e que necessitam de assistência multiprofissional (fisioterapia, musicoterapia, terapia ocupacional, psicologia, nutrição, dentre outras) ${ }^{7}$. Este serviço proporciona a melhora da autoestima, da saúde física e mental, estimula a independência e autonomia, retarda o aparecimento de doenças limitantes e evita depressão e isolamento social? .

Apesar da Política Nacional do Idoso prever a implementação de 'centros-dia', em grande parte do Brasil não existem instituições públicas para idosos, e as existentes são de caráter particular.

No que se refere ao atendimento da pessoa com deficiência intelectual no processo de envelhecimento, a situação é ainda mais complexa, pois a inexistência de serviços públicos especializados leva à situação de vulnerabilidade e sobrecarga as famílias cuidadoras. Observa-se que estes serviços são escassos e comumente organizados pela sociedade civil, como os disponibilizados pela Associação de Pais e Amigos dos Excepcionais (Apae).

Grande parte das Apaes além de prestar atendimentos reabilitatórios também é responsável pela permanência dessa pessoa nos Centros de Convivência ou outros serviços adequados ao seu atendimento.

Os 'centros de convivência' da Apae se assemelham ao 'centros-dia' na sua funcionalidade, dando suporte não somente ao aluno, mas também prestando orientação e apoio à família cuidadora, tendo como proposta oferecer atendimento sócio ocupacional a pessoa com deficiência intelectual acima de 35 anos, em processo de envelhecimento precoce ou idosa, oportunizando atividades que possibilitem condições para aquisição e/ ou manutenção das habilidades funcionais e sociais básicas de independência.

Sendo assim, diante de tal realidade, emergiu o seguinte questionamento: Qual a conformação de um serviço de 'centro-dia' para a pessoa envelhecente com deficiência intelectual? Consoante, objetivou-se mapear os serviços, fluxos de atendimento e organização do serviço do 'centro-dia' das Apaes da Grande Florianópolis para o atendimento de pessoa envelhecente com deficiência intelectual. 
Justifica-se a relevância do presente estudo, uma vez que o mapeamento dos serviços e seus componentes, permitirá aos profissionais de saúde maior compreensão quando a conjuntura do serviço de 'centro-dia' no que concerne ao indivíduo idoso com deficiência intelectual, culminando em novas perspectivas para a melhoria da qualidade de vida e inclusão social destes atores.

\section{Metodologia}

Trata-se de uma pesquisa de abordagem qualitativa, exploratório-descritiva realizada nas Apaes da região da Grande Florianópolis, de março a abril de 2015. Conforme o Instituto Brasileiro de Geografia e Estatística ${ }^{\mathbf{8}}$ (IBGE) a região da Grande Florianópolis é composta por 22 municípios, sendo que, de acordo com a Associação de Pais e Amigos dos Excepcionais9 apenas 17 possuem Apae.

Para tanto, realizou-se pesquisa documental nos sites oficiais das Apaes para investigação do Projetos Políticos Institucionais (PPI). No caso da instituição que não ofereceu tal ferramenta virtual foi realizado contato telefônico e digital com o coordenador pedagógico e/ou diretor da instituição, solicitando fornecimento dos dados para responder ao roteiro semiestruturado elaborado pelas autoras.

Os resultados foram agrupados por semelhança de ideias centrais, conforme o método de Análise Temática de Minayo ${ }^{10}$. No que diz respeito à discussão, esta foi embasada, à luz da Teoria da Modificabilidade Estrutural de Reuven Feuerstein conforme descrito por Da $\operatorname{Ros}^{11}$ e literatura pertinente.

Quanto aos aspectos éticos foi respeitada a Resolução n ${ }^{\circ} 466 / 2012$ que dispõe sobre os procedimentos éticos em pesquisa com seres humanos. Cabe pontuar, que o presente estudo é parte integrante do macroprojeto 'A rede de atenção à saúde e de suporte social à pessoa idosa com deficiência na grande Florianópolis e as tecnologias de cuidado', sob parecer 24410513.5.0000.0121 do Comitê de Ética da Universidade Federal de Santa Catarina (UFSC).

\section{Resultados e discussão}

O processo de envelhecimento das pessoas com deficiência intelectual acontece de modo atípico e precoce, com comprometimentos em suas habilidades cognitivas, sensoriais, adaptativas, afetivas e sociais, podendo alterar sua autonomia e independência.

$\mathrm{O}$ IBGE $^{8}$ informa que 45 milhões de pessoas disseram ter alguma deficiência (24\%), dos quais 2.611 .536 com deficiência intelectual. Santa Catarina apresenta uma população total de 6.727 .148 habitantes e o número de pessoas com deficiência intelectual chega a 71.956 (1\%). Na região da Grande Florianópolis, de 1.111.082 habitantes, 11.597 (1\%), são classificados como pessoas com deficiência intelectual. No levantamento realizado nos 17 municípios da Região da Grande Florianópolis que possuem Apae, há 1802 alunos institucionalizados, dos quais 465 (25,8\%) são pessoas envelhecentes com deficiência intelectual acima de 35 anos.

Apesar de fazerem parte da Federação Nacional das Apaes, cada instituição possui sua organização interna, assim como sua diferenciada proposta de atendimento às pessoas com deficiência intelectual, seguindo as diretrizes que fundamentam cada modalidade de atendimento na sua especificidade. Os serviços que podem ser oferecidos nas Apaes organizam-se da seguinte forma: a) Estimulação Essencial (EE): para crianças de zero a 3 anos e 11 meses que previne e atua sobre o atraso no desenvolvimento neuropsicomotor visando desenvolver seu potencial; b) Serviço Atendimento Educacional Especializado (Saede): visa atender os educandos com Diagnóstico de Deficiência Mental e/ou Transtorno do Espectro do Autismo (TEA), frequentando etapas e modalidades da Educação 
Básica; c) Serviço Pedagógico Específico Ocupacional (SPE): educandos na faixa etária acima de 17 anos com diagnóstico de deficiência mental com severos comprometimentos cognitivos, associado ou não a outras deficiências e/ou transtorno global do desenvolvimento com baixa funcionalidade; d) Educação Profissional (EP) (faixa etária a partir dos 14 anos): capacita o educando para o exercício de atividades profissionais que possibilitem acesso ao mundo do trabalho, promovendo independência e autonomia nos aspectos individuais e sociais; e) Oficina Protegida Terapêutica (OPT): oferecida aos adolescentes e adultos que devido ao grau de deficiência, transitória ou permanente, não possam desempenhar atividade laboral no mercado competitivo de trabalho; f) Centro de Convivência (CC): tem como proposta oferecer atendimento sócio- ocupacional a pessoa com deficiência intelectual acima de 35 anos, em processo de envelhecimento precoce ou idosa ${ }^{\mathbf{1 2}}$.

Nessa perspectiva, das 17 Apaes apenas duas possuem o atendimento de 'centro de convivência' e o Serviço Pedagógico Específico (SPE). Oito utilizam apenas o SPE, quatro oferecem o serviço de Educação Profissional (EP) e duas utilizam Oficinas Protegidas Terapêuticas (OPT).

Após análise dos fluxos de atendimentos e organização dos serviços destas instituições, mediante a análise de conteúdo temática ${ }^{10}$, emergiram duas categorias temáticas, quais sejam:

\section{Centro-dia: definindo conceitos}

Para Da Ros"1 todo ser humano é modificável, desde que submetido a uma interação humana, planejada e sistematizada para esse objetivo: as Experiências de Aprendizagem Mediada. Sob esta ótica, destaca-se que os fatores cognitivos, afetivos e socioculturais determinam diferencialmente o desenvolvimento da pessoa, numa ação dinâmica e dialética ${ }^{13}$.

Atualmente há uma importante mudança drástica no perfil das pessoas com deficiência intelectual que envelhecem em todo o mundo, o que reforça a necessidade de conscientização dos serviços voltados a essa população ${ }^{\mathbf{1 4}}$.

Ainda, são pressupostos do 'centro-dia' exercitar o processo de autogestão e autodefensoria. Para a Federação Nacional das Associações de Pais e Amigos dos Excepcionais ${ }^{9}$, trata-se de uma área que valoriza e capacita a pessoa com deficiência intelectual e sua família, dando-lhes vez e voz, incentivando sua participação ativa no movimento com o intuito de orientá-los e capacitá-los para se tornarem protagonistas de seus direitos e deveres; promovendo atividades ocupacionais, lúdicas e de lazer que possam beneficiar a saúde física e mental; possibilitar Atividades da Vida Diária (AVD), desenvolvendo os sentidos remanescentes, mediante atividades funcionais contextualizadas.

Ainda que seja possível encontrar respaldo científico sobre o envelhecimento da pessoa com deficiência intelectual são poucas as contribuições sobre a execução de serviços direcionados a esse público específico, o que evidencia a necessidade de construção de um campo profissional que promova a interlocução entre a teoria e a prática desenvolvida $^{15}$. O atendimento adequado deve levar em consideração os aspectos da senilidade, fatores implícitos ao cuidado gerontológico.

\section{Estruturação e organização pedagógica}

Para se configurar um 'centro-dia' há alguns critérios de elegibilidade relacionados à pessoa que o frequentará, a estrutura, organização e equipe de atendimento.

Os critérios de elegibilidade da pessoa que o frequentará comporta: adultos com 
diagnóstico de deficiência intelectual com idade cronológica igual ou superior a 35 anos com sinais de envelhecimento precoce; apresentar semi-independência nas atividades da vida diária (higiene, vestuário e alimentação); não apresentar perspectivas de inserção no mundo do trabalho e não apresentar transtornos de comportamento que interfiram na proposta de atendimento.

Em relação à estrutura, há de se considerar que a literatura sugere, que esta deva ter adaptações nos banheiros, mobílias, portas, rampas, corrimão, piso antiderrapante, entre outros, tais como espaços destinados à proposta dos atendimentos, cozinha funcional para as atividades de vida diária, salas ambientes para confecção de artesanatos, espaço de jogos, leitura e atividades pedagógicas, assim como um pátio para atividades externas ${ }^{16}$.

Já a equipe multiprofissional deve ser composta pelos profissionais das áreas de serviço social, fonoaudiologia, fisioterapia, coordenação pedagógica, professor, psicologia, terapia ocupacional, informática, artes, educação física, dança, médico psiquiatra e neurologista.

No entanto, a Portaria n ${ }^{\circ} 835$ de 25 de abril de $2012^{17}$, prevê para um Centro Especializado de Reabilitação (CER), cuja equipe deve ser minimamente composta por médico, fisioterapeuta, fonoaudiólogo, terapeuta ocupacional, assistente social e enfermeiro.

Em relação ao planejamento pedagógico, este é guiado por um caderno pedagógico, através de propostas anuais divididas em planejamentos mensais, seguindo a orientação da coordenação pedagógica.

O processo de avaliação pedagógica acontece de forma processual e contínua, através de observações e registros que possibilitem traçar novas metas e objetivos de maneira a qualificar o atendimento através de ações planejadas. Toda intervenção pedagógica deve ser organizada pelo planejamento pedagógico, sendo que este deve conter atividades intencionadas, significativas e transcendentes. Toda proposta de trabalho deve ser repassada às famílias cuidadoras, na intenção de que estas deem continuidade aos cuidados e estímulos em suas casas.

Para acessar o serviço de 'centro-dia', a família cuidadora (ou seu representante legal) deverá buscar o atendimento especializado pessoalmente para agendar o processo de triagem. Na triagem, havendo a necessidade, encaminha-se para a avaliação que apresentará o parecer diagnóstico e indicará sua elegibilidade ou não para o atendimento. Uma vez elegível, a pessoa frequenta durante meio turno o 'centro-dia'. No contra turno, preconiza-se a possibilidade de buscar alternativas de inclusão na sociedade.

Considerando tais resultados, propõe-se que o 'centro-dia' seja pautado na legislação vigente, quais sejam: a Política Nacional do Idoso $^{6}$, o Estatuto do Idoso', o Sistema Único de Assistência Social e a Declaração dos Direitos das Pessoas Portadoras de Deficiências ${ }^{3}$.

\section{Conclusões}

Este estudo possibilitou conhecer o quantitativo de pessoas envelhecentes, com deficiência intelectual num município da região sul do País, bem como a estruturação e funcionamento de 'centros-dia', um dispositivo imprescindível para o atendimento destas pessoas.

As leis já existentes com a intenção de atender gratuitamente estas pessoas precisam ser cumpridas e monitoradas, não deixando somente a cargo das instituições especializadas como as Apaes fazerem sozinhas esse papel, que é do poder público. Acima de tudo, pensar no atendimento à pessoa envelhecente com deficiência intelectual é promover seu empoderamento e a oportunidade de inclusão social, oferecendo-lhe uma vida digna e cidadã.

\section{Colaboradores}

Bonatelli LCS, contribuiu substancialmente para a concepção, coleta, análise e 
interpretação dos dados do trabalho. Schier J, contribuiu substancialmente para a concepção, planejamento, análise e interpretação dos dados e participou da aprovação da versão final do manuscrito. Girondi JBR, contribuiu substancialmente para a concepção e o planejamento ou para a análise e a interpretação dos dados; contribuiu significativamente na elaboração do rascunho ou revisão crítica do conteúdo; e participou da aprovação da versão final do manuscrito. Hammerschmidt KSA, contribuiu significativamente na elaboração do rascunho ou revisão crítica do conteúdo; e participou da aprovação da versão final do manuscrito. Tristão FR, contribuiu significativamente na revisão crítica do conteúdo e participou da aprovação da versão final do manuscrito.

\section{Agradecimentos}

\author{
À Fundação de Amparo à Pesquisa e Inovação \\ do Estado de Santa Catarina (Fapesc) pelo fi- \\ nanciamento da pesquisa.
}

\section{Referências}

1. Pimenta RA, Rodrigues LA, Greguol M. Avaliação da qualidade de vida e sobrecarga de cuidadores de pessoas com deficiência intelectual. R Bras Ci Saúde [internet]. 2010 [acesso em 2018 jul 21]; 14(3):6976. Disponível em: http://www.luzimarteixeira. com.br/wp-content/uploads/2010/12/artigo_rbcs. pdf.

2. Brasil. Lei no 10741, de 2003. Dispõe sobre o Estatuto do Idoso e dá outras providências. Diário Oficial da União. 1 Out 2003.

3. Paraná. Ministério Público do Paraná. Resolução ONU n ${ }^{\circ} 2542 / 75$. Declaração do Direito das Pessoas Portadoras de Deficiência. Diário Oficial da União. 1975.

4. Bousfield RMF. Centro dia para idosos como uma possibilidade à permanência no convívio familiar: uma contribuição do serviço social [monografia]. Palhoça: Universidade do Sul de Santa Catarina; 2010. 103 p.
5. Brasil. Decreto $n^{\circ} 1.948$, de 3 de julho de 1996. Aprova a Política Nacional do Idoso. Diário Oficial da União. 3 Jul 1996.

6. Brasil. Portaria $n^{\circ} 184 / 20101$ de julho de 2010 . Aprova a Política Nacional de Promoção da Saúde. Diário Oficial da União. 1 Jul 2010.

7. Neris MSM. Atendimento em centro-dia para idosos em situação de dependência: alternativa de serviço da política nacional de assistência social? [dissertação]. Rio de Janeiro: Fiocruz; 2011. 146 p.

8. Instituto Brasileiro de Geografia e Estatística. Municípios de Florianópolis [internet]. Rio de Janeiro: IBGE; 2018 [acesso em 2018 jul 22]. Disponível em: http://cidades.ibge.gov.br/xtras/uf.php?lang=\&cod $\mathrm{uf}=42 \&$ search $=$ santa-catarina.

9. Federação Nacional das Associações de Pais e Amigos dos Excepcionais. Autogestão e auto defensoria: conquistando autonomia e participação [inter- 
net]. Brasília, DF: Fenapaes; 2009 [acesso em 2018 jul 24]. Disponível em: https://apaebrasil.org.br/fl/ normal/5144-livro_autodefensoria_final.pdf.

10. Minayo MCM. O desafio do conhecimento: pesquisa qualitativa em saúde. São Paulo: Hucitec; 2010.

11. Ros SZ. Pedagogia e mediação em Reuven Feuerstein: o processo de mudança em adultos com história de deficiência. São Paulo: Plexus; 2002.

12. São Paulo. Apae [internet]. São Paulo: Apae; 2018 [acesso em 2018 jul 22]. Disponível em: http:// www.apaesp.org.br/pt-br/Paginas/default.aspx.

13. Ron RRD. Aprendizagem Mediada. Rev Eletr Educ Tecnol SENAI-SP [internet]. 2011 jun [acesso em 2018 jul 21]; 5(10)1:25. Disponível em: https://carlaechabe.files.wordpress.com/2013/11/aprendizagem-mediada.pdf.

14. Maccarron M, Reilly E. Supporting persons with intellectual disability and dementia: quality dementia care standards: A guide to practice. Dublin: Daughters of Charity Service; 2010.

15. Fonseca LM, Gonçalves AS, Carvalho CL et al. Implicações sobre deficiência intelectual e longevida- de: projeto piloto de intervenção em casos de senilidade. Fenapaes [internet]. 2013 maio-ago [acesso em 2018 jul 21]; 1(2)3:21. Disponível em: https:// www.researchgate.net/publication/280556842.

16. Guerreiro EMBR. A acessibilidade e a educação: um direito constitucional como base para um direito social da pessoa com deficiência. Rev Educ Espec [internet]. 2012 [acesso em 2018 jul 24]; 25(43)217:232. Disponível em: https://periodicos.ufsm.br/index. php/educacaoespecial/article/viewFile/4415/3816.

17. Brasil. Portaria $n^{\circ} 835$, de 25 de Abril de 2012. Institui incentivos financeiros de investimento e de custeio para o Componente Atenção Especializada da Rede de Cuidados à Pessoa com Deficiência no âmbito do Sistema Único de Saúde. Diário Oficial da União. 25 Abr 2012. [acesso em 2018 set 18]. Disponível em: http://bvsms.saude.gov.br/bvs/saudelegis/gm/2012/prt0835_25_04_2012.html.

Recebido em 27/12/2017

Aprovado em 31/07/2018

Conflito de interesses: inexistente

Suporte financeiro: Fundação de Amparo à Pesquisa e Inovação

do Estado de Santa Catarina (Fapesc) 